\title{
ELEMENTOS CLAVE DE LA FINANCIACIÓN COMUNITARIA DEL DESARROLLO URBANO:
}

LA SUPERACIÓN DE LA METODOLOGÍA DE LAS INICIATIVAS URBAN

\author{
María-Rosario Alonso-lbáñez \\ Catedrática de Derecho Administrativo \\ Coordinadora Urban Red
}

En consonancia con las Conclusiones del Consejo Europeo del 17 de junio de 2010, en las que se adoptó la Estrategia Europa 2020 para un crecimiento inteligente, sostenible e integrador ${ }^{1}$, la Unión Europea y los Estados miembros están comprometidos desde entonces a hacer lo necesario para conseguir un crecimiento económico en los términos que allí quedaron explicitados. A tales efectos, en el actual período de programación 2014-2020 los Fondos Estructurales y de Inversión Europeos (EIE) se han diseñado para que los Estados miembros tengan que centrar sus prioridades de inversión en línea con los 5 objetivos europeos que a largo plazo fija la citada Estrategia: la investigación y la innovación; el cambio climático y la energía; el empleo; la educación; y la reducción de la pobreza. En correspondencia con estas prioridades, los Fondos EIE deben centrar su ayuda en un número limitado de objetivos temáticos comunes, definidos en el art. 9 del Reglamento (UE) 1303/2013 del Parlamento Europeo y del Consejo de 17 de diciembre de $2013^{2}$, objetivos todos ellos

\footnotetext{
${ }^{1}$ Comunicación de la Comisión "Europa 2020. Una estrategia para un crecimiento inteligente, sostenible e integrador", COM (2010) 2020 final, de 3 de marzo de 2010.

${ }^{2}$ Reglamento (UE) 1303/2013 del Parlamento Europeo y del Consejo de 17 de diciembre de 2013 por el que se establecen disposiciones comunes relativas al Fondo Europeo de Desarrollo Regional, al Fondo
} 


\section{WPSReview International on Sustainable}

\section{Housing and Urban Renewal (RI-SHUR)}

que deben perseguirse en el marco de un desarrollo sostenible, tal como se recoge en el artículo 11 y el artículo 191, apartado 1, del TFUE. Cada Fondo EIE tiene a su vez sus propios objetivos o prioridades de inversión. Unos y otros han de traducirse en objetivos y prioridades nacionales y regionales. Los Estados deben llevar a sus respectivos Programas Nacionales de Reformas los objetivos temáticos que seleccionen y combinen, y a los Programas Operativos las prioridades de inversión de cada Estado.

Cada Programa Operativo tiene sus correspondientes Ejes prioritarios, que son en última instancia las actuaciones concretas a ejecutar en cada Comunidad Autónoma, promovidas a nivel nacional o a nivel regional. Esto es lo que ha venido sucediendo así hasta la fecha. Sin embargo, el actual periodo de programación ha establecido importantes novedades, con clara repercusión en el medio urbano, que son en las que quisiera centrarme en este momento:

Así, resulta necesario aplicar "enfoques integrados" sobre la entera política de cohesión. A los enfoques estratégicos se da contenido a través de los distintos objetivos temáticos y los ejes prioritarios de los distintos Programas Operativos. Cada Estado deberá promover actuaciones donde queden comprometidas las autoridades

Social Europeo, al Fondo de Cohesión, al Fondo Europeo Agrícola de Desarrollo Rural y al Fondo Europeo Marítimo y de la Pesca, y por el que se establecen disposiciones generales relativas al Fondo Europeo de Desarrollo Regional, al Fondo Social Europeo, al Fondo de Cohesión y al Fondo Europeo Marítimo y de la Pesca, y se deroga el Reglamento (CE) 1083/2006 del Consejo (DOUE L 347/320, de 20 de diciembre de 2013. Identifica los siguientes 11 objetivos temáticos comunes:

1) potenciar la investigación, el desarrollo tecnológico y la innovación;

2) mejorar el uso y la calidad de las tecnologías de la información y de la comunicación y el acceso a las mismas;

3) mejorar la competitividad de las pyme;

4) favorecer la transición a una economía baja en carbono en todos los sectores;

5) promover la adaptación al cambio climático y la prevención y gestión de riesgos;

6) conservar y proteger el medio ambiente y promover la eficiencia de los recursos;

7) promover el transporte sostenible y eliminar los estrangulamientos en las infraestructuras de red fundamentales;

8) promover la sostenibilidad y la calidad en el empleo y favorecer la movilidad laboral;

9) promover la inclusión social y luchar contra la pobreza y cualquier forma de discriminación;

10) invertir en educación, formación y formación profesional para la adquisición de capacidades y un aprendizaje permanente;

11) mejorar la capacidad institucional de las autoridades públicas y las partes interesadas y la eficiencia de la administración pública.

WPS RI-SHUR, n6, 2017, vol.2, ISSN: 2387-1768

MONOGRÁFICO SOBRE LAS ESTRATEGIAS DE DESARROLLO URBANO

SOSTENIBLE E INTEGRADO (EDUSI) 


\section{WPSReview International on Sustainable}

\section{Housing and Urban Renewal (RI-SHUR)}

regionales y locales, dotándolas de mayores funciones en los procesos de toma de decisiones y ejecuciones. El Acuerdo de Asociación que cada Estado miembro firme con la Comisión deberá especificarlo. En particular, deberá indicar "un enfoque integrado del desarrollo territorial apoyado por los Fondos EIE o un resumen de los enfoques integrados del desarrollo territorial basado en el contenido de los programas operativos" $^{3}$-y que, debe destacarse, no es fácil de encontrar el Acuerdo de Asociación de España ${ }^{4}$.

Estos enfoques integrados debieran quedar garantizados a través de la potenciación de la participación local (a través de Estrategias de Desarrollo Local Participativo CLLD-, basadas en la experiencia del LEADER en las zonas rurales) y de Inversiones Territoriales Integradas (ITI), que es otra de las novedades que se han introducido en la actual reglamentación de la política de cohesión ${ }^{5}$. En todo caso, requisito previo del enfoque integrado será asegurar la cooperación entre los distintos niveles administrativos, la sociedad civil, la economía local y los ciudadanos.

Por su parte, a fin de lograr específicamente un "enfoque de desarrollo integrado" mediante la utilización de los Fondos EIE, coordinados con otros instrumentos y políticas de la UE, un Marco Estratégico Común (MEC) deberá proporcionar, con

\footnotetext{
${ }^{3}$ Lo que se traduce en la necesidad de establecer en los citados Acuerdos de Asociación, de conformidad con el art. 15.2.a) del Reglamento (UE) 1301/2013: "i) las medidas que garanticen un enfoque integrado del uso de los Fondos EIE para el desarrollo territorial de zonas concretas subregionales, acompañadas de los principios que determinen las zonas urbanas en las que deban ejecutarse acciones integradas para un desarrollo urbano sostenible. ii) los principales ámbitos prioritarios para la cooperación en virtud de los Fondos EIE, habida cuenta, cuando proceda, de las estrategias macrorregionales y de las estrategias de las cuencas marítimas; iii) cuando proceda, un enfoque integrado para abordar las necesidades específicas de las zonas geográficas más afectadas por la pobreza o de grupos destinatarios que corren mayor riesgo de discriminación o exclusión social, prestando especial atención a las comunidades marginadas, a las personas con discapacidad, a los desempleados de larga duración y a los jóvenes que no trabajan, estudian ni siguen ninguna formación; iv) cuando proceda, un enfoque integrado para abordar los retos demográficos de las regiones o las necesidades específicas de las zonas geográficas que padecen desventajas naturales o demográficas graves y permanentes a tenor del artículo 174 del TFUE".

ES/ipr/fcp1420/p/pa/Documents/20141022 AA spain 20142020 .pdf http://www.dgfc.sepg.minhafp.gob.es/sitios/dgfc/es-

${ }^{5} A$ las ITI se refiere el artículo 36 del Reglamento (UE) 1303/2013, a desarrollar mediante un programa operativo específico o mediante un eje prioritario específico, de conformidad con el artículo 96, apartado 1, párrafo primero, letra c), del Reglamento (UE) 1303/2013.
} 


\section{WPSReview International on Sustainable}

\section{Housing and Urban Renewal (RI-SHUR)}

arreglo al artículo 10 del Reglamento UE 1303/2013, los principios rectores estratégicos.

No obstante, es el FEDER el instrumento que apoyará, mediante sus propios Programas Operativos, el enfoque de desarrollo integrado aplicado al desarrollo valga la redundancia- urbano sostenible, "a través de estrategias que establezcan medidas integradas para hacer frente a los retos económicos, ambientales, climáticos, demográficos y sociales que afectan a las zonas urbanas, teniendo en cuenta al mismo tiempo la necesidad de promover los vínculos entre el ámbito urbano y el rurap' ${ }^{\prime}$.

Los artículos 7 y 8 del Reglamento (UE) 1301/2013 del Parlamento Europeo y del Consejo de 17 de diciembre de 2013 acogen un apoyo decidido al desarrollo "sostenible" e "integrado" en el medio urbano: Al menos un $5 \%$ de los recursos del FEDER asignados a nivel nacional en virtud del objetivo temático "inversión en crecimiento y empleo" se asignarán a "medidas integradas para el desarrollo urbano sostenible", en las que las ciudades, en particular, las "autoridades urbanas" -los organismos subregionales o locales responsables de la aplicación de "estrategias urbanas sostenibles" serán los encargados de las tareas relacionadas, por lo menos, con la selección de las operaciones, de conformidad con el artículo 123, apartado 6, del Reglamento (UE) 1303/2013 o, según corresponda, de conformidad con el artículo 123, apartado 7, de dicho Reglamento.

El carácter integrado, como parte del "acervo urbano" ${ }^{7}$, junto con la proyección de la sostenibilidad al desarrollo urbano (aunque inicialmente muy ligado a una concepción ambientalista de la sostenibilidad), eran las dos ideas clave que venían impregnando

\footnotetext{
${ }^{6}$ Artículo 7.1 del Reglamento (UE) 1301/2013 del Parlamento Europeo y del Consejo de 17 de diciembre de 2013 sobre el Fondo Europeo de Desarrollo Regional y sobre disposiciones específicas relativas al objetivo de inversión en crecimiento y empleo y por el que se deroga el Reglamento (CE) 1080/2006 (DOUE L 347/289, de 20 de diciembre de 2013).

"Para una aproximación clara a la evolución del "acervo urbano europeo" cfr. E. De Santiago Rodríguez, "El proceso de construcción de la Agenda Urbana de la Unión Europea: de la Declaración de Toledo al Pacto de Amsterdam", en S. De Gregorio Hurtado (Coord.) Urban regeneration in the UE, TRIA 18 (1/2017), pp.23-46.
} 


\section{WPSReview International on Sustainable}

\section{Housing and Urban Renewal (RI-SHUR)}

hasta el actual periodo de programación toda la política urbana europea. La Carta de Leipzig (2007) vinculó las dos ideas como requisito para alcanzar con éxito la sostenibilidad de las ciudades, quedando así acuñado el concepto "desarrollo urbano sostenible e integrado" (DUSI). La Declaración de Toledo y su Documento de referencia sobre regeneración urbana integrada (2010) vinieron posteriormente a dotar de un protocolo común de entendimiento del enfoque integrado aplicado al desarrollo urbano $^{8}$, viniendo ahora la reglamentación del FEDER a facilitar la aplicación del citado enfoque introduciendo la necesidad de disponer de una "Estrategia" de ciudad coherente, equilibrada y con una visión de largo plazo -las EDUSI-, contemplando como específicas herramientas de gestión integrada -las ITI y el CLLD-, fortaleciendo a las "autoridades urbanas", y aumentando el nivel de la experimentación en este campo, a través de las llamadas "acciones Urbanas Innovadoras" (AUI) ${ }^{9}$, a través de las cuales se podrán apoyar todas las actividades necesarias para alcanzar los objetivos temáticos comunes contemplados en el artículo 9.1 del Reglamento (UE) 1303/2013 y las prioridades de inversión correspondientes establecidas en el artículo 5 del Reglamento (UE) $1301 / 2013^{10}$. En este contexto, la EDUSI, que tiene un contenido muy reglamentado, se caracteriza por ser:

\footnotetext{
${ }^{8}$ Al respecto, puede verse M.R. Alonso lbáñez, "De la Política Urbanística a la Política Urbana: Los Retos Actuales del Derecho Urbanístico", Revista de Derecho Urbanístico y Medio Ambiente, 277, 2012, pp. 1327.

${ }^{9}$ De conformidad con el artículo 8 del Reglamento (UE) 1301/2013, a iniciativa de la Comisión, el FEDER podrá apoyar medidas innovadoras en el ámbito del desarrollo urbano sostenible, de conformidad con el artículo 92, apartado 8, del Reglamento (UE) no 1303/2013. Entre esas medidas se incluirán estudios y proyectos piloto para identificar o probar soluciones nuevas en respuesta a cuestiones que están relacionadas con el desarrollo urbano sostenible y que son de interés para la Unión. La Comisión fomentará la participación de los socios pertinentes a que se refiere el artículo 5 , apartado 1 , del Reglamento (UE) no 1303/2013 en la preparación y ejecución de acciones innovadoras.

${ }^{10}$ Son las siguientes: 1) potenciar la investigación, el desarrollo tecnológico y la innovación; 2) mejorar el acceso, el uso y la calidad de las tecnologías de la información y de la comunicación; 3) mejorar la competitividad de las pymes; 4) favorecer el paso a una economía de bajo nivel de emisión de carbono en todos los sectores; 5 ) promover la adaptación al cambio climático y la prevención y gestión de riesgos; 6) conservar y proteger el medio ambiente y promover la eficiencia de los recursos, contemplando específicamente las acciones "para mejorar el entorno urbano y revitalizar las ciudades"; 7) promover el transporte sostenible; 8) promover la sostenibilidad y la calidad en el empleo y favorecer la movilidad laboral; 9) promover la inclusión social y luchar contra la pobreza y cualquier discriminación; 10) invertir en educación, formación y formación profesional para la adquisición de capacidades y el aprendizaje permanente, mediante el desarrollo de las infraestructuras de educación y formación; 11) mejorar la capacidad institucional de la administración pública y las partes interesadas y la eficiencia de la
}

WPS RI-SHUR, n6, 2017, vol.2, ISSN: 2387-1768

MONOGRÁFICO SOBRE LAS ESTRATEGIAS DE DESARROLLO URBANO

SOSTENIBLE E INTEGRADO (EDUSI) 


\section{WPSReview International on Sustainable \\ Housing and Urban Renewal (RI-SHUR)}

- Un documento, concreto y sistemático, que se elabora anticipadamente para programar y dirigir las actuaciones previstas.

- Tener un carácter estratégico, lo que implica una reflexión a largo plazo sobre la entera ciudad.

- Define prioridades, lo que supone una elección y una jerarquización de retos a abordar y de objetivos a alcanzar.

- Tener un componente territorial, es decir, se desarrolla sobre un territorio con actuaciones o proyectos urbanos concretos

- Debe integrar las diferentes aproximaciones sectoriales: físicas, ambientales, urbanísticas, económicas, sociales, culturales, etc.

Con la EDUSI se supera claramente la metodología de las anteriores iniciativas URBAN, basadas en un enfoque "area-based", de intervenciones sobre áreas concretas especialmente delimitadas, pasándose a financiar estrategias a nivel de ciudad, tomando en consideración la complejidad de formas urbanas en que ésta hoy se desenvuelve.

España ha aprobado en el actual periodo de programación, entre otros, el Programa Operativo de Crecimiento Sostenible 2014-2020 ${ }^{11}$, concentrando en cuatro ejes prioritarios, más uno de asistencia técnica, dirigidos a cubrir las necesidades identificadas en las siguientes temáticas: Eje 4: Economía baja en Carbono; Eje 12: Desarrollo urbano integrado y sostenible; Eje 6: Calidad del agua; Eje 7: Transporte sostenible, y Eje 13: Asistencia Técnica. Al Eje 12 se ha llevado el desarrollo del art. 7 del Reglamento (UE) 1301/2013, destinando más de 1.000 millones de euros para el desarrollo de EDUSIs en las ciudades o áreas funcionales urbanas de más de 20.000

administración pública mediante medidas de refuerzo de las capacidades institucionales y de la eficiencia de las administraciones públicas y de los servicios públicos relacionados.

11 http://www.dgfc.sepg.minhafp.gob.es/sitios/dgfc/esES/ipr/fcp1420/p/Prog Op Plurirregionales/Documents/PO CrecimientoSostenible FEDER 20142020.pdf

WPS RI-SHUR, n6, 2017, vol.2, ISSN: 2387-1768

MONOGRÁFICO SOBRE LAS ESTRATEGIAS DE DESARROLLO URBANO

SOSTENIBLE E INTEGRADO (EDUSI) 


\section{WPSReview International on Sustainable}

\section{Housing and Urban Renewal (RI-SHUR)}

habitantes. La primera convocatoria, ya finalizada, distribuyó el $70 \%$ de los fondos ${ }^{12}$. En una segunda convocatoria se distribuyó el $30 \%$ restante ${ }^{13}$. Una tercera convocatoria, con aportación de fondos adicionales, está pendiente de resolución ${ }^{14}$.

\footnotetext{
12 Orden HAP/2427/2015, de 13 de noviembre, por la que se aprueban las bases y la primera convocatoria para la selección de estrategias de Desarrollo Urbano Sostenible e Integrado que serán cofinanciadas mediante el programa operativo FEDER de crecimiento sostenible 2014-2020. Mediante la Resolución de 29 de septiembre de 2016, de la Secretaría de Estado de Presupuestos y Gastos, se conceden las ayudas de la primera convocatoria para la selección de estrategias de Desarrollo Urbano Sostenible e Integrado que serán cofinanciadas mediante el Programa Operativo FEDER de crecimiento sostenible 2014-2020 (BOE 3 de octubre 2016).

${ }^{13}$ Orden HAP/1610/2016, de 6 de octubre, por la que se aprueba la segunda convocatoria para la selección de estrategias de Desarrollo Urbano Sostenible e Integrado que serán cofinanciadas mediante el Programa Operativo de Crecimiento Sostenible FEDER 2014-2020. Mediante Resolución de 18 de mayo de 2017, de la Secretaría de Estado de Presupuestos y Gastos, se conceden ayudas de la segunda convocatoria (BOE 22 de mayo 2017).

${ }_{14}$ Orden HFP/888/2017, de 19 de septiembre, por la que se modifica la Orden HAP/2427/2015, de 13 de noviembre, por la que se aprueban las bases y la primera convocatoria para la selección de estrategias de Desarrollo Urbano Sostenible e Integrado que serán cofinanciadas mediante el programa operativo FEDER de crecimiento sostenible 2014-2020; y por la que se aprueba la tercera convocatoria para la selección de las citadas estrategias.
}

WPS RI-SHUR, no6, 2017, vol.2, ISSN: 2387-1768

MONOGRÁFICO SOBRE LAS ESTRATEGIAS DE DESARROLLO URBANO

SOSTENIBLE E INTEGRADO (EDUSI) 